\title{
A Mirror Representation of Others' Actions in the Human Anterior Parietal Cortex
}

\author{
Lior Shmuelof ${ }^{1}$ and Ehud Zohary ${ }^{1,2}$ \\ ${ }^{1}$ Department of Neurobiology and ${ }^{2}$ Interdisciplinary Center for Neural Computation, Hebrew University, Jerusalem, Israel 91904
}

\begin{abstract}
The anterior part of the human intraparietal sulcus is known to be involved in visually guided grasping. This region is also active during the observation of object manipulation by others. Here, we explore the nature of action representation using functional magnetic resonance imaging (fMRI). Fourteen subjects observed video clips showing object manipulation by the right or left hand. The clips were presented in either the right or left peripheral visual field. The fMRI activation in the occipital cortex and in the caudal sections of the parietal cortex was specific to the visual-field location of the clips. In contrast, the principal factor determining the response in anterior intraparietal cortex was the identity of the observed hand. Furthermore, these "hand-specific" parietal areas also showed contralateral hand specificity during self action (i.e., object manipulation) without visual feedback. A similar selectivity for the identity of the observed hand was seen when using a region of interest analysis, focusing on individually defined visuomotor voxels within the parietal cortex. This dual visuomotor grasping representation lends further evidence for the existence of a mirror system in humans and suggests that the anterior intraparietal cortex is involved in the specific motor simulation of hand actions.
\end{abstract}

Key words: fMRI; vision; motor; object manipulation; action observation; mirror system

\section{Introduction}

Evidence from recent years suggests that some cortical motor areas (i.e., engaged in motor planning and execution), are also active during mere viewing of others' actions (Gangitano et al., 2001; Buccino et al., 2004; Nelissen et al., 2005). The first clear evidence for this was the discovery of mirror neurons in the macaque monkey's frontal lobe (area F5) and inferior parietal lobule (Gallese et al., 1996; Rizzolatti et al., 1996; Fogassi et al., 2005). These neurons discharge when the monkey executes goaldirected actions, such as grasping, even when the action is executed in the dark. Importantly, they are also active during observation of another monkey, or the experimenter, performing a similar action.

According to the direct matching hypothesis (DMH) raised by Rizzolatti and colleagues (Iacoboni et al., 1999; Rizzolatti et al., 2001), observation of actions made by others should be manifested in a change in the excitability of the observer's motor elements that match the ones that are seen. One way to assess this is by measuring the magnitude of motor-evoked potential (MEP), as a result of applying transcranial magnetic stimulation (TMS) to the motor cortex. Indeed, Gangitano et al. (2004) have shown that passive observation of object-grasping video clips amplifies the magnitude of the MEP resulting from the TMS pulse, in concordance with the kinematic profile of the observed action. In a functional magnetic resonance imaging (fMRI) study, Buccino

Received May 1, 2006; revised Aug. 9, 2006; accepted Aug. 10, 2006.

This work was supported by the Israel Science Foundation of the Israel Academy of Sciences Grant 80009. We thank Tanya Orlov for the help with the 3D cortex reconstructions. We thank Tamar Makin and Alit Stark for helpful discussions.

Correspondence should be addressed to Ehud Zohary, Department of Neurobiology and Interdisciplinary Center for Neural Computation, Hebrew University, Jerusalem, Israel 91904. E-mail: udiz@lobster.Is.huji.ac.il. DOI:10.1523/JNEUROSCI.1836-06.2006

Copyright $\odot 2006$ Society for Neuroscience $\quad$ 0270-6474/06/269736-07\$15.00/0 et al. (2001) have studied patterns of activation in the posterior parietal cortex (and the premotor cortex) during observation of object manipulation by the foot, hand, or mouth. They found that the evoked fMRI activation in these areas was according to the somatotopic mapping principles of the motor cortex (i.e., the human homunculus).

The anterior intraparietal cortex is known to be active during grasping movements in both monkeys (Murata et al., 2000) and humans (Culham et al., 2003; Frey et al., 2005). Imaging studies in humans have shown that this cortex is also active during visually elicited motor imagery (Parsons et al., 1995; Bonda et al., 1996; de Lange et al., 2005). We have recently shown (Shmuelof and Zohary, 2005) that viewing video clips of object manipulation results in a robust activation along the intraparietal sulcus (IPS). Interestingly, the anterior parietal activation showed contralateral preference for the location of the hand (rather than the object) and was specific to the grasping component of the action. This dual grasping-specific activity during both action execution and action observation suggests that this region may be part of the putative human mirror system. However, because the right hand was displayed mainly in the right visual field (and the left hand in the left field), we could not determine whether the anterior parietal preference was attributable to the fact that the hand was seen in the contralateral field (visual-field representation) or that the viewed acting hand was the contralateral one (suggesting an internal motor representation of the viewed action). We demonstrate here that in anterior intraparietal cortex, external actions are represented according to the encoding principles of the motor system (Penfield and Rasmussen, 1952), regardless of the location of the actions in the visual field. Second, we show that the anterior intraparietal cortex is active both during action observation and action execution (without visual feedback) using a specific body part. 


\section{Materials and Methods}

Subjects. Fourteen volunteers without a history of neurological, psychiatric, or visual deficits (seven women and seven men, 25-35 years of age) participated in the fMRI experiments. The Tel-Aviv Sourasky Medical Center Ethics Committee approved the experimental procedure. A written informed consent was obtained from each subject.

MRI acquisition. The blood oxygenation level-dependent (BOLD) fMRI measurements were performed in a whole-body 1.5 T, Signa Horizon, LX8.25 General Electric (Fairfield, CT) scanner. The functional MRI protocols were based on a multislice gradient echoplanar imaging and a standard head or surface coil. The functional data were obtained under the optimal timing parameters. For experiment 1 (visual experiment): repetition time (TR), $3 \mathrm{~s}$; echo time (TE), $55 \mathrm{~ms}$; flip angle, $90^{\circ}$; imaging matrix, $64 \times 64$; field of view (FOV), $21 \mathrm{~cm}$. The 29 slices with slice thickness of $4 \mathrm{~mm}$ (with no gap) were oriented in the axial position. The scan covered the whole brain.

The somatomotor mapping was conducted using the following parameters: TR, $2 \mathrm{~s}$; TE, $55 \mathrm{~ms}$; flip angle, $90^{\circ}$; imaging matrix, $64 \times 64$; FOV, $21 \mathrm{~cm}$. The 19 slices with slice thickness of $4 \mathrm{~mm}$ (with $1 \mathrm{~mm}$ gap) were oriented in an oblique axis. The scan covered most of the cortex except for the most anterior parts of the frontal lobe. The fMRI images were superimposed on T1-weighted three-dimensional (3D) SPGR images (spatial resolution, $1 \times 1 \times 1 \mathrm{~mm}$ ).

Experimental setup. The video clips were taken using a digital camera (TRV60E; Sony, Tokyo, Japan), edited on a personal computer (using the program Windows Movie Maker), and projected via a liquid crystal display projector (MP 7200; Epson, Nagano, Japan) onto a tangent screen located inside the scanner in front of the subject. The subjects viewed the screen through a tilted mirror.

\section{Main experiment}

A set of 16 object-manipulation epochs, $12 \mathrm{~s}$ long, and 2 visual control epochs were shown in this experiment. Each epoch of right-hand footage (in the left and right periphery) was composed of 10 clips (900-1500 ms each), of a right hand approaching from the right, grasping and releasing an object (total time, $12 \mathrm{~s}$ ). The clips were taken using a set of 15 manmade graspable objects, such as different jars, cups, scissors, etc. Six to 10 clips were taken with each object, showing different grasping movements. Each epoch was composed of 10 clips of different objects. Lefthand clips were generated by a "flip horizontal" operation on the "righthand" clips. In all clips, the objects remained stationary and did not move throughout the grasping movements. In the control "scramble" conditions, a 12 s object-manipulation epoch was decomposed to frames (30 frames a second), every frame was spatially scrambled $(36 \times 24$ fragments in each frame, ensuring that the hand and the objects could no longer be identified), and the frames were then recomposed to a $12 \mathrm{~s}$ epoch. A fixation point appeared in the middle of the screen throughout the experiment. The clips were centered $16.5^{\circ}$ to the right or the left of the fixation point and subtended $20 \times 15^{\circ}$.

\section{Experimental paradigm}

The experiment was performed using a block design format. Six blocks were interleaved and were repeated four times, with different stimuli, in a counterbalanced manner. Each block lasted $12 \mathrm{~s}$, followed by a blank period of $9 \mathrm{~s}$. The first and last blank periods were longer (21 and $15 \mathrm{~s}$, respectively). Subjects were trained before the experiment to make sure that they maintained fixation and understood the instructions.

The conditions (Fig. 1) involved viewing the following scenes: (1) object manipulations by the right hand, shown in the left visual field; (2) object manipulation by the right hand, shown in the right visual field; (3) object manipulation by the left hand, shown in the left visual field; (4) object manipulation by the left hand, shown in the right visual field; (5) spatially scrambled version of the objectmanipulation clips, shown in the left visual field; and (6) spatially scrambled version of the object-manipulation clips, shown in the right visual field. The "scramble" control conditions (numbers 5 and 6 above) were introduced to allow selection of voxels showing differential activation during the observation of the object-manipulation clips (compared with the scrambled versions).

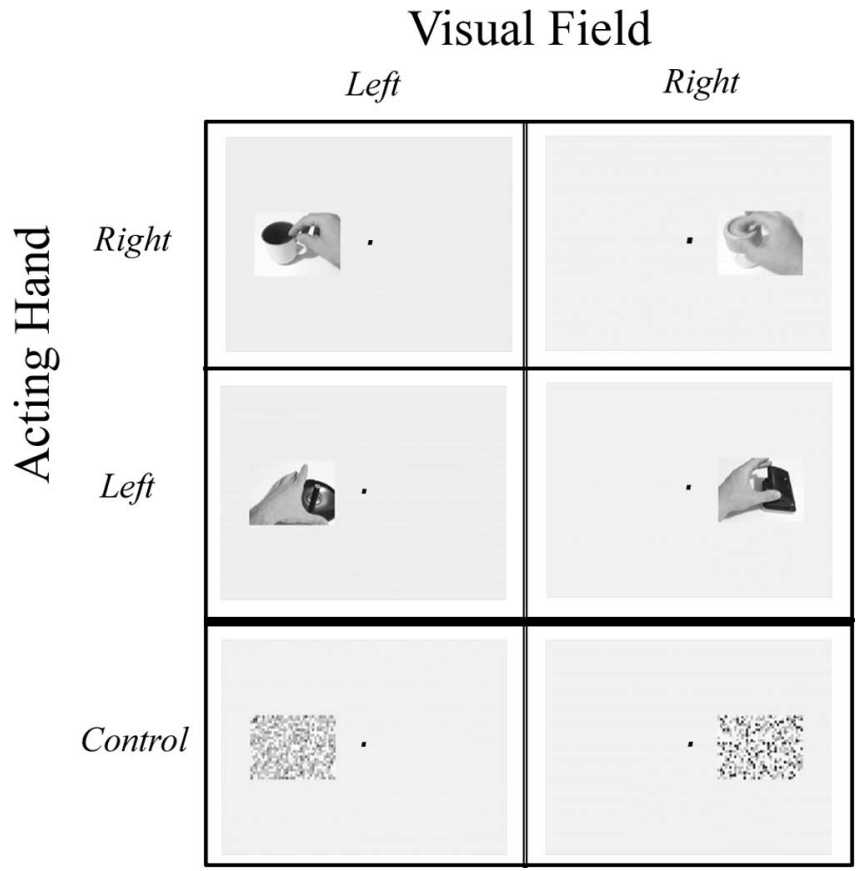

Figure 1. Experimental conditions. Six conditions were interleaved in a block design experiment. In four of them, the subjects observed a single hand approaching and grasping different objects. Two variables were manipulated in the experiment: hand identity (left or right; top and middle rows) and visual field [the location of the clips on the screen (left or right periphery, columns)]. In addition, we included two visual control conditions, in which a spatially scrambled version of the objectmanipulation clips was presented in the left or right periphery (bottom row). The subjects were required to maintain fixation on a central dot throughout the experiment.

\section{Somatomotor mapping}

We also conducted a somatomotor localizer experiment in which the subjects were required to move their left or right hand, foot, or mouth, according to oral instructions. The words were recorded by a digital recording device using GoldWave Shareware software (GoldWave, St. John's, Newfoundland, Canada). The instructions were $1 \mathrm{~s}$ long and consisted of the name of the body part to be moved (right/left hand, right/left foot, mouth) or a "stop" instruction. The execution instructions were presented in the last $1 \mathrm{~s}$ of the rest block, and the "stop" signal was given in the last second of the specific epoch.

The five conditions included (1) left-hand movements, (2) right-hand movements (in both cases subjects manipulated a cube with their hand), (3) flexion and extension of the left ankle, (4) flexion and extension of the right ankle, and (5) movement of the tongue along the teeth. All conditions were interleaved and repeated 4 times. Each block lasted $12 \mathrm{~s}$ followed by a blank period of $10 \mathrm{~s}$. The first and last blank periods were longer (20 and $14 \mathrm{~s}$, respectively). Subjects were trained before the experiment to make sure that they understood the commands and maintained fixation. The subjects focused on a fixation point at the center of the screen throughout the experiment. Because of the supine posture of the subjects, they could not see their actions.

\section{Data analysis}

Data analysis was performed using the BrainVoyager 4.96 and BrainVoyager Qx software packages (Brain Innovation, Maastricht, The Netherlands). For each subject, the two-dimensional (2D) functional data were aligned to $2 \mathrm{D}$ anatomical slices of the same subject. Before statistical analysis, raw data were examined for motion and signal artifacts. Head motion correction and high-pass temporal smoothing in the frequency domain (3 cycles/total scan time) were applied to remove drifts and to improve the signal-to-noise ratio. The complete data set was transformed into Talairach space, $Z$-normalized and concatenated. A general linear model (GLM) approach was used to generate statistical parametric maps. The hemodynamic response function was modeled using parameters such as those used by Boynton et al. (1996). Significance levels were 
calculated, taking into account the probability of a false detection for any given cluster, by requiring that statistically significant activation is seen in contiguous groups of voxels. The implemented method is based on the approach of Forman et al. (1995) and accomplished by Monte Carlo simulations (using a BV QX plug-in).

Across-subjects statistical parametric maps (Figs. 2, 6) were calculated using a hierarchical random-effects model analysis (Friston et al., 1999). The cortical surface was reconstructed from the 3D SPGR scan. The procedure included segmentation of the white matter using a growregion function, the smooth covering of a sphere around the segmented region, and the expansion of the reconstructed white matter into the gray matter. The surface was then unfolded, cut along the calcarine sulcus and flattened.

\section{Region-of-interest analysis}

The single-subject visuomotor regions of interest (ROIs) were identified in the parietal lobes based on conjunction analysis between visual object-manipulation areas and motor hand areas (Fig. 3a). Visual object-manipulation areas were identified as the voxels showing significantly higher activation during observation of the hand action clips than during observation of a spatially scrambled version of those clips $(p<0.01$, cluster-size correction). Motor hand areas were identified as the voxels showing significantly higher activation $(p<0.001$, clustersize correction) during object manipulation by the contralateral hand than during contralateral foot movements.

The activation time course of individual subjects was obtained from each ROI, using a GLM approach. The time course was pooled across subjects to yield the average activation time course (Fig. 4a). Similarly, the average percentage signal change (from 9 to $15 \mathrm{~s}$ after the onset of the condition) was calculated for each subject and then averaged across subjects to yield the bar histograms (Figs. 4b, 5). A paired two-tailed $t$ test was applied to test for significant differences between conditions.

\section{Results}

In experiment 1 , we investigate the patterns of brain activation during observation of object-manipulation clips, presented in the periphery. The goal of this experiment was to differentiate between brain areas that show preference to stimuli presented in the contralateral visual field [a pattern of representation that is characteristic throughout the visual system (Engel et al., 1996)] and those that showed preference for the contralateral acting hand [a feature that characterizes motor system brain areas (Penfield and Rasmussen, 1952)]. Note that in all conditions, the subjects viewed the clips without making any grasping movements. Therefore, areas sensitive to the identity of the viewed acting hand are likely to be candidates for having an internal motor representation of external observed actions.
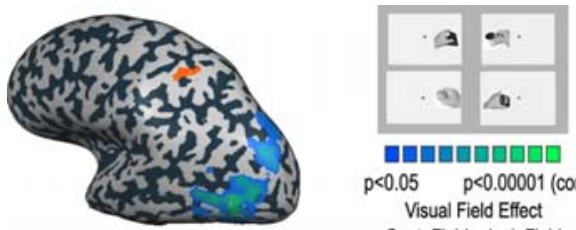

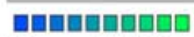
$p<0.05 \quad p<0.00001$ (corr.) Visual Field Effect Cont. Field $>$ Ipsi. Field

Left Hemisphere

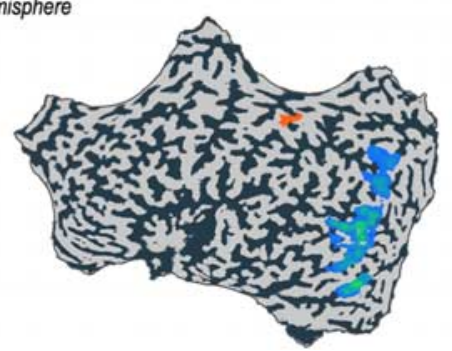

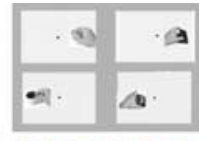

뭄ㅁㅁㅁㅁㅁㅁำ $p<0.05 \quad p<0.00001$ (corr.) Hand-Identity Effect Cont. Hand > Ipsi. Hand

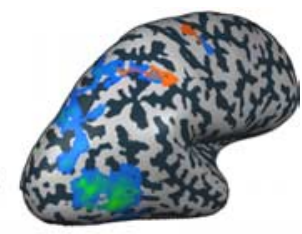

Right Hemisphere

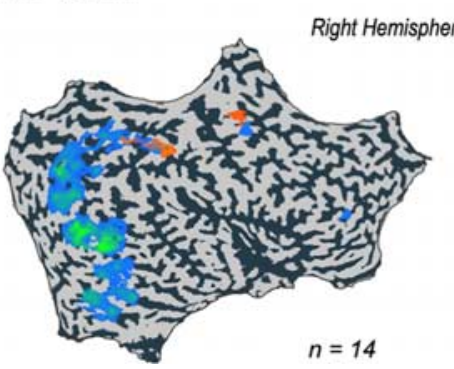

Figure 2. Visual-field versus hand-identity effects. A statistical parametric map of the group results (14 subjects), using a random-effect GLM analysis and cluster-size correction (corr.) for multiple comparisons. Red-to-yellow colors depict handidentity areas, i.e., voxels demonstrating increasingly significant preference for clips showing the contralateral hand action compared with the ipsilateral (Ipsi.) hand action. Blue-to-green colors indicate areas showing a visual-field effect, i.e., voxels showing increasingly significant preference for object-manipulation clips presented in the contralateral field than in the ipsilateral field. Significant hand-identity effects can be seen bilaterally in the superior bank of the aIPS and the superior parietal lobule, as well as in the precentral sulcus of the right hemisphere. Significant visual-field effects can be seen throughout the object-selective occipital cortex bilaterally, in the caudal section of the right parietal cortex, and in the right precentral sulcus.

a.
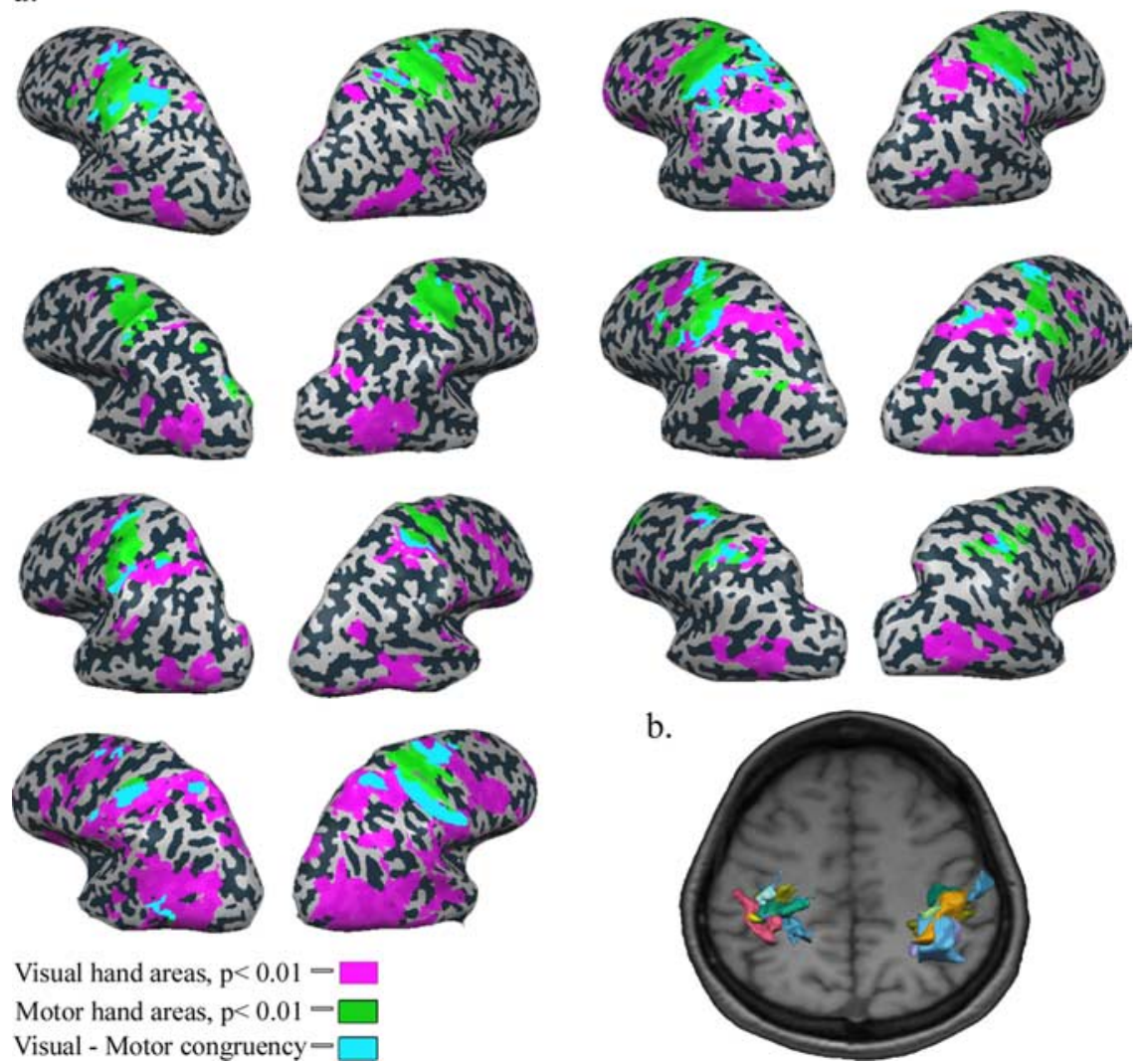

Motor hand areas, $\mathrm{p}<0.01-$

Visual - Motor congruency

$y=\square$

Figure 3. Identifying visuomotor areas: single-subject analysis. $\boldsymbol{a}$, Inflated cortical maps of seven of the nine subjects that participated in the motor mapping experiment. Visual hand action areas (purple) were identified as voxels showing significantly higher activation during observation of the hand action clips than during observation of a spatially scrambled version of those clips. Motor hand areas (green) were identified as the voxels showing significantly greater activation during object manipulation using the contralateral hand compared with contralateral ankle movement. Voxels showing both visual and motor preference for hand actions are depicted in cyan. $\boldsymbol{b}$, Superposition of the 3D structure of the visuomotor ROIs of each of the nine subjects overlaid on a representative horizontal slice of the cortical sheet. Each subject's ROl is depicted in a different color. Note the spatial congruency between the subjects' visuomotor ROIs in the anterior intraparietal cortex. 
a.
Left Hemisphere

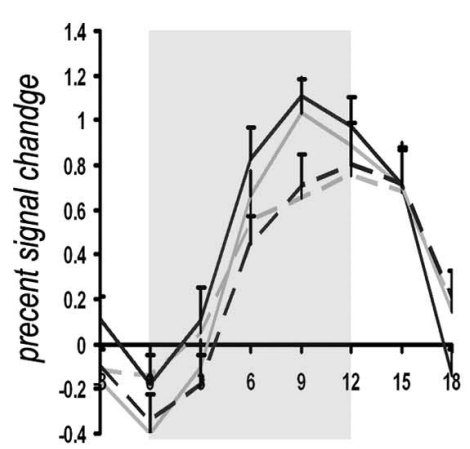

Right Hemisphere

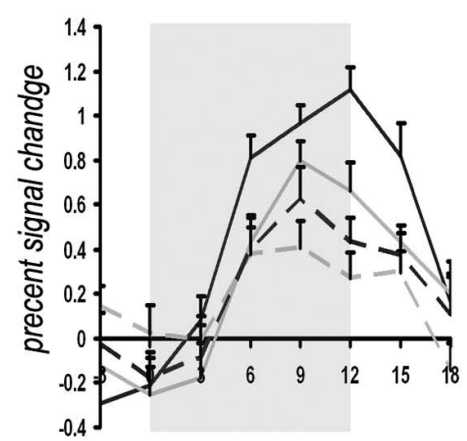

cont. Hand cont. Field

cont. Hand ipsi. Field

ipsi. Hand cont. Field . - - - - -

ipsi. Hand ipsi. Field b. Hand effect

Field effect
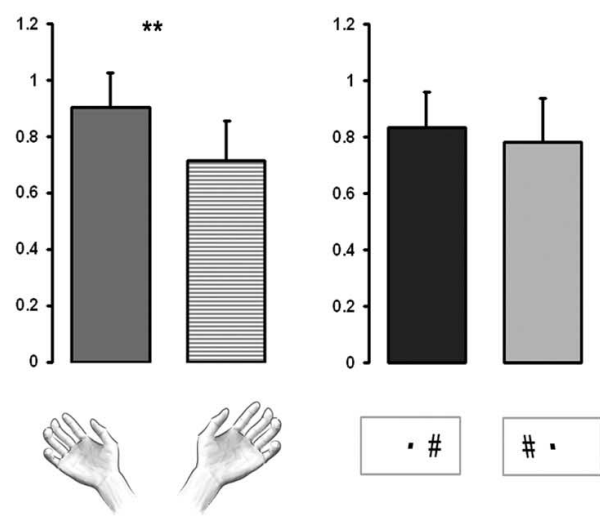

- \#
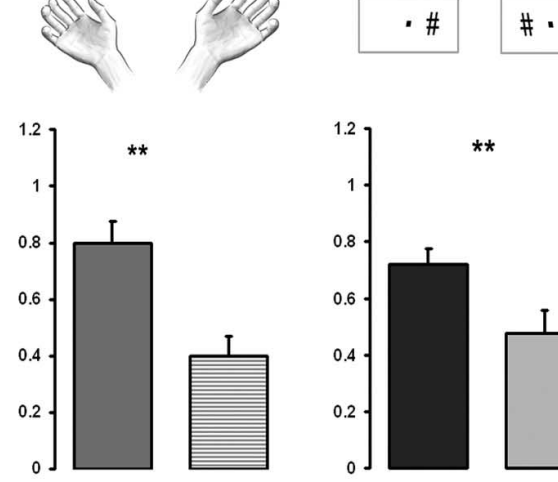

cont. Hand ipsi. Hand
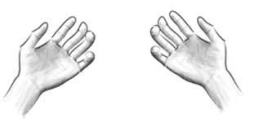

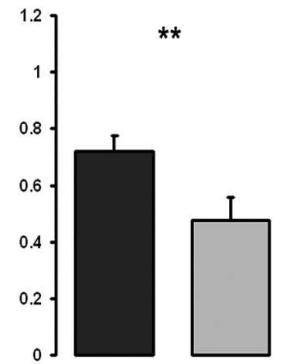

cont. Field ipsi. Field

\#.
- \#
Figure 4. Hand-identity and visual-field effects: ROl analysis. $\boldsymbol{a}$, Averaged hemodynamic response curves for the four experimental conditions in the visuomotor ROIs of the anterior intraparietal cortex $(n=9)$. Error bars denote the SEM. The conditions are specified separately to each of the two hemispheres, according to its contralateral (cont.) or ipsilateral (ipsi.) viewed hand, as well as visual field. $\boldsymbol{b}$, Bar histograms of the two effects, hand identity and visual field, plotted separately for each hemisphere, showing the averaged activation (across all subjects) for each of the factors (cont./ipsi. viewed hand, cont./ipsi. visual field, icons below indicate the visual condition). Error bars denote the SEM. Asterisks denote significance $\left(^{* *} p<0.01\right)$. Significant visual-field effect can be seen in the right anterior intraparietal ROI, whereas significant hand-identity effects can be seen bilaterally, in both ROls.
To differentiate between a visualoriented and a motor-oriented representation, we used a two-by-two factorial design; the first variable was the identity of the seen acting hand (left or right), and the second variable was the location of the viewed objects (and grasping hand) in the peripheral visual field (right or left of the fixation point). We expected that the dominant factor in the visual areas of occipitotemporal cortex would be the visual-field position. Our main focus, however, was in areas showing a hand-identity effect: having a greater BOLD signal when viewing the contralateral acting hand than when viewing the ipsilateral hand, regardless of the location of the stimuli in the visual field (for example, viewing the right-hand manipulating objects should generate greater activation in the left hemisphere than in the right one). Because contralateral hand preference is a typical characteristic of motor areas, such mirror activity, in the absence of motor behavior by the subject, is consistent with an internal motor representation of the observed actions.

\section{Group analysis}

Figure 2 depicts the brain areas that display significant visual-field and/or handidentity effects, using a random-effect analysis and correction for multiple comparisons. Cortical areas showing significantly higher BOLD activation during observation of clips in the contralateral visual field than in the ipsilateral field are depicted in a green-to-blue scale. Contralateral visual-field preference can be seen in both hemispheres, throughout the occipitotemporal cortex, as well as in dorsal areas, in the transverse occipital sulcus. In addition, in the right hemisphere, we
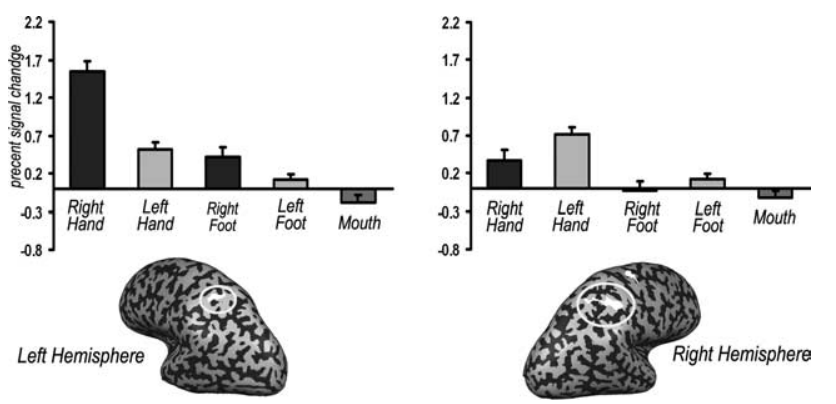

Figure 5. Analysis of fMRl activation in the parietal "hand-identity" areas during the somatomotor mapping. An analysis of the fMRI signal in the areas that show a clear dependence on the viewed hand identity in the group analysis (shown in the bottom and in Fig. 2) during the somatomotor mapping. Averaged activation $(n=9)$ during the somatomotor mapping experiment in which the subjects moved their right or lefthand, foot, or mouth in the dark. Errorbars denote SEM. Significant preference can be seen for actions of the contralateral hand compared with the other body parts and the ipsilateral hand (paired, two-tailed $t$ test; left ROI: right hand $>$ left hand, $p<0.0002$; right ROI: right hand $<$ left hand, $p<0.005$ ). Thus, the parietal regions that are sensitive to the identity of the viewed hand show a similar contralateral specificity for the acting hand. found a significant preference for left-field clips in the parietal lobe, in the caudal intraparietal cortex and in the superior parietal lobule. Cortical areas that displayed a significant hand-identity effect, (i.e., preference to video clips showing the contralateral hand) are depicted in a yellow-to-red scale. These are mainly found between the superior bank of the anterior intraparietal sulcus (aIPS) and the postcentral gyrus (the anterior intraparietal cortex).

To summarize, we replicated the well known contralateral visual-field representation in the occipitotemporal cortex. More importantly, we found evidence for a different type of representation, in which the dominant factor determining the level of activity is the identity of the viewed acting hand (in the absence of the subject's own hand action), in the anterior intraparietal cortex.

\section{Identifying visuomotor areas: ROI analysis}

The results of the group analysis are consistent with the idea of an internal motor representation of others' actions in the anterior intraparietal cortex. However, if this activation is indeed an internal motor representation of someone else's action (i.e., part of the mirror system), the same areas ought to be selectively active during motor action of the same body part. We therefore per- 
formed another experiment, mapping the patterns of activation elicited by action using various body parts without visual feedback (see Materials and Methods). Next, we identified (in each of the 9 subjects that participated in this experiment) the visuomotor areas as those voxels that show both (1) selective activation during object-manipulation observation (all objectmanipulation epochs $>$ scramble epochs, $p<0.01$, cluster-size correction) and (2) hand-specific motor activation $(p<0.001$, cluster-size correction). Hand-specific motor activation was defined by contrasting the activation during object manipulation using the contralateral hand with the activation during contralateral ankle movement. Figure $3 a$ presents the motor hand areas (green), the object-manipulation observation areas (purple), and the conjunction between the areas (visuomotor areas, cyan) in seven of the nine subjects that participated in both experiments (the conjunction between the visual and motor areas of the remaining two subjects was evident in the $2 \mathrm{D}$ slice views, but could not be seen clearly on their reconstructed surface maps). Figure $3 b$ shows the superposition of the 3D structure of the ROIs of all nine subjects overlaid on a representative horizontal slice. Each subject's ROI (selected on the basis of their combined visual \& motor selectivity) is depicted in a different color. One can appreciate that the ROIs in the parietal cortex are located roughly in the same region, within the anterior section of the intraparietal cortex (left anterior intraparietal cortex: average size, $2792 \pm 685$ $\mathrm{mm}^{3}$; right anterior intraparietal cortex: average size, $1711 \pm 454$ $\left.\mathrm{mm}^{3}\right)$.

We further investigated whether the visual properties of the separately defined visuomotor ROIs are consistent with the direct matching hypothesis, i.e., that external actions are represented on the basis of the motor properties of the viewed action (in our case, according to the identity of the acting hand). Therefore, we compared the fMRI activation in the four different objectmanipulation clips. Note that the ROIs were selected on the basis of their visual preference for the object-manipulation clips (as well as their motor hand selectivity), but no preference was given for one object-manipulation viewing condition over the other. Thus, we could compare the fMRI activation elicited during the four different object-manipulation viewing conditions to examine the effect of the two factors (visual field and hand identity) in the two visuomotor ROIs.

We find that in the left anterior intraparietal cortex, the greatest fMRI activation occurred during viewing of the contralateral (right) hand grasping objects, regardless of the grasping scene's location in the visual field. This suggests that hand identity was the main factor in determining the fMRI activation in this ROI. In the right anterior intraparietal cortex, the greatest fMRI activation was for viewing the contralateral (left) hand in the contralateral (left) visual field, whereas the weakest BOLD signal was elicited when viewing the ipsilateral hand in the ipsilateral visual field. This suggests that both hand identity and visual field play a role in the level of activation in this ROI. Indeed, a two-factor ANOVA showed a significant hand-identity effect in the left anterior intraparietal cortex $\left(F_{(1,8)}=6.7, p<0.032\right)$. In the right anterior intraparietal cortex, both hand-identity and visual-field effects were significant, although the effect of hand identity was more pronounced (hand identity: $F_{(1,8)}=23, p<0.001$; visual field: $\left.F_{(1,8)}=11.3, p<0.01\right)$. No interaction between the two effects was found in the two ROIs. Note that this analysis matches the hand-identity effect in the anterior intraparietal cortex, presented in the group analysis (Fig. 2). In fact, close inspection of the group analysis map in Figure 2 shows the same qualitative difference between the two hemispheres (i.e., preference for the contralateral hand in the left anterior intraparietal cortex and to both the contralateral hand and visual field in the right anterior intraparietal cortex).

\section{Motor properties in "hand-identity areas"}

The results from the ROI analysis indicate that regions within the anterior intraparietal cortex have both visual and motor properties. Furthermore, in these regions, the fMRI activation evoked by the observed hand actions depends on the identity of the acting hand. This result is in accordance with the direct-matching hypothesis, stating that seeing action performed by others invokes an internal motor representation of the same action.

To test this issue further, from another angle, we looked at the specific motor properties of cortical areas that showed a preference for the contralateral hand viewed actions [in the group analysis (Fig. 5, inset, same as orange clusters in Fig. 2)]. If seeing action performed by others evokes an internal motor representation of the same hand, we would expect to find activation in these areas during motor behavior, using the same hand, even without any visual feedback. Indeed, the fMRI activation (in the areas that showed visual hand-identity specificity in the anterior intraparietal cortex) is clearly greater for hand movement compared with the other body parts (i.e., foot or mouth movements). Furthermore, in both hemispheres, there is an obvious and statistically significant preference for motor actions using the contralateral hand over the ipsilateral one [paired two-tailed $t$ test; $p<0.005$ in the right hemisphere; $p<0.001$ in the left hemisphere (Fig. 5a)].

\section{Grasp-viewing-based adaptation}

We have previously demonstrated (Shmuelof and Zohary, 2005) that certain areas in the anterior intraparietal cortex show grasp-viewing-based adaptation: repetitive observation of the same object grasping movement leads to a reduction of the BOLD signal (compared with observation of different grasping movements). To give further evidence that the activation of the "hand-identity" ROIs may be viewed as classical "mirror activity," we tested whether these regions also show grasp-viewing-based adaptation. The fMRI signal in these group-based ROIs (selected on the basis of their preference for the contralateral hand viewed actions) clearly shows graspviewing-based adaptation (paired two-tailed $t$ test; $p<0.002$ in the right anterior intraparietal and $p<0.001$ in the left anterior intraparietal, $n=14$; object-based adaptation not significant in the two ROIs), suggesting that they are also sensitive to the fine details of the viewed action.

Finally, we studied the degree of overlap between the (group-based) cortical areas showing viewed-hand preference, hand motor properties (in the current study) and the cortical areas that show grasp-viewing-based adaptation. Note that although the video clips were projected in different locations in the two studies (central visual field in the previous adaptation study, peripheral in the current one), there is a striking congruency between the two loci (Fig. 6).

Interestingly, a large proportion of the areas that show congruency between the two visual experiments, showing a handidentity effect and a grasping-based adaptation, are also selectively active during movement of the contralateral hand. This once again suggests that observed hand actions are represented in the anterior intraparietal cortex according to implicit motor principles, which are very different from the known rules of representation in the visual cortex. 


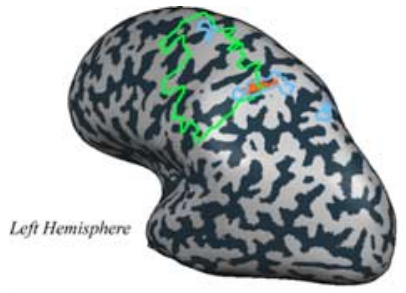
Cont. Hand $>$ Cont. Foot (p<0.05, n=9, corr.)
Grasp vicwing Adaptation ( $p 00.05, \mathrm{n}=14$, corr.) -

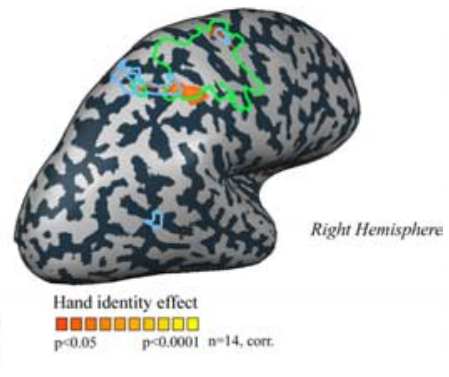

Figure 6. Congruency maps with the "hand-identity areas." Correspondence between the cortical areas that show significant hand-identity effect in the group analysis (red to yellow; $n=14$ ), cortical areas that show grasp-viewing-based adaptation in a group analysis (circled in cyan; $n=14$ ), and cortical areas that show preference for contralateral hand action (over contralateral foot movement) in the group analysis (circled in light green; $n=9$ ). Physical overlap between the mapped cortical areas is limited to the anterior intraparietal hand-identity areas (in both hemispheres), as well as the right precentral sulcus.

\section{Discussion}

This study supports the idea of a visuomotor system that is involved in the perception of external actions. First, we demonstrate that regions within the superior bank of the aIPS are sensitive to the identity of the observed hand (right or left) more than to its location in the visual field. The same parietal regions are also selectively active during execution of hand actions without visual feedback (but not foot or tongue movements). These characteristics are consistent with the $\mathrm{DMH}$ raised by Rizzolatti et al. (1996, 2001). However, before discussing the possible utility of such a mirror system, we first consider the possibility that the observed fMRI activation during the above conditions was attributable to overt mimicry of the observed actions, because it may have aided the subjects to perform the task (requiring them to judge whether the action was the same or different from the previous one). According to such a scheme, the presumed contralateral representation of the observed hand is in fact a representation of an action of which the subjects are not aware. Although we cannot rule out this explanation entirely, we think it is unlikely because if the subjects actually moved their hand (despite the instruction to refrain from doing so) we would expect to find activation in primary motor cortex [around the central sulcus; similar to the activation found in the somatomotor mapping experiment (Fig. 6)]. This clearly was not the case.

\section{Hand and field effects in the parietal cortex}

The parietal cortex is known to be engaged in visuomotor transformations, which are necessary for visually guided actions. However, the nature of the assumed transition from a visual representation of the target objects to a motor representation of our actions toward them is still largely unknown. One way to track this transition is to study the effects of disruption of normal parietal function on action performance, focusing on the importance of the visual (target visual-field location) versus motor (acting hand) aspects of the task. Virtual lesions (using TMS) in the intraparietal sulcus lead to the disruption of path correction during reaching with the contralateral hand (but not the ipsilateral one) toward a moving target (Desmurget et al., 1999). Analogously, inactivation of the monkey AIP (using muscimol) leads to deficits in the preshaping of the contralateral hand toward the final grasping movement (Gallese et al., 1994). Finally, patients with damage involving the aIPS have problems in adjusting the aperture of the fingers during grasping with the contralateral hand (Binkofski et al., 1998).
In contrast, patients suffering from optic ataxia as a result of damage to more posterior sections of the intraparietal sulcus, close to the occipitoparietal junction (Karnath and Perenin, 2005) show impairments in reaching for an object (with either of the two hands) under visual guidance, when the object is positioned in the contralesional visual periphery, but not when it is placed in the ipsilesional visual field. The relative contributions of the position of the target (in the visual field) and the acting hand identity (left or right) during reaching movements were also assessed in a PET study. The results revealed a hand-identity effect (during action) in the superior parietal lobule of the two hemispheres and in the inferior parietal lobule of the right hemisphere (Kertzman et al., 1997).

Interestingly, in our study, observation of video clips, without any action, generated a similar pattern of activation in the posterior parietal cortex, whereas the activation in the most anterior section of the parietal cortex was consistent with a representation that is based on the motor characteristics of the actions (handidentity specificity), the representation in the caudal sections of the parietal cortex was based on the visual properties of the clips.

\section{Functional divisions within the mirror system}

Accumulating evidence demonstrates that action observation in humans activates a complex network of cortical regions in the parietal, frontal, and temporal lobes (Iacoboni et al., 1999; Buccino et al., 2001, 2004; Gangitano et al., 2001; de Lange et al., 2005; Aziz-Zadeh et al., 2006; Cross et al., 2006). However, the most frequently mentioned areas comprising the putative "human mirror system" are the anterior part of the inferior parietal lobule (IPL), as well as the precentral gyrus and the posterior part of the inferior frontal gyrus (IFG). In our study, observation of the video clips elicited significant activation (with respect to the scrambled control clips) in the same general locations (see supplemental Fig. 1, presenting the cortical activation for objectmanipulation observation, available at www.jneurosci.org as supplemental material). Nevertheless, the activation in the IPL and IFG did not show specificity for the viewed hand. The lack of viewed hand specificity in these areas may result from their sensitivity to other elements of the observed action, such as the meaning of the action (which is clear in gestures), its complexity, or to the task of the observer (Molnar-Szakacs et al., 2005). Such attributes do not usually depend on the identity of the viewed hand.

The locus of hand-identity-related activation in our study (i.e., showing greater activation for the viewed contralateral hand than the ipsilateral one) was between the superior bank of the aIPS and the postcentral sulcus (left hemisphere: $x,-35, y,-45$, $z$, 58; right: $33,-43,57)$. This locus is adjacent to the grasping specific focus reported by Culham et al. (2003), (showing significantly greater activation during visually guided grasping than during reaching; left hemisphere $-38,-48,52$; right $40,-50$, 50) and to a locus reported by Iacoboni et al. (1999), showing specific increase in fMRI activation for finger movement that result from imitation (compared with finger movement according to spatial cues; right anterior parietal cortex: 37, -40, 57). Furthermore, Tunik et al. (2005) have recently shown (using TMS) that virtual lesions to the human anterior parietal cortex cause errors in reach-to-grasp kinematics toward a rotating target only immediately after $(65 \mathrm{~ms})$ target rotation. Interestingly, if the target remains stationary, the TMS pulse to the aIPS does not cause significant errors in the adjustment of the grasp movements (to fit the target size). These results may indicate that the aIPS is involved in integrating an efference copy of the motor commands with incoming visual inputs (i.e., predicting the visual outcome of the motor action), to guide hand actions. This interpretation 
may also explain the unique visuomotor representation in the anterior intraparietal cortex, showing clear hand specificity, because the motor commands and expected outcome are obviously dependent on the acting hand. Additional research is obviously required to elucidate the division of labor within areas comprising the putative human mirror system.

\section{Laterality effects}

Although we demonstrate a symmetric representation of hand identity in the right and left anterior parietal cortex, both the group results and the ROI analysis reveal a convergence of the hand-identity and visual-field effects only in the right anterior intraparietal cortex. Interestingly, we found a similar convergence of "ventral" object-oriented representation and "dorsal" grasping-oriented representation in the right anterior parietal cortex, but not in the left hemisphere, in a recent study that mapped cortical areas showing grasp viewing-based adaptation and object viewing-based adaptation (Shmuelof and Zohary, 2005). This asymmetry is also consistent with behavioral experiments, showing that precision grasping using the left hand (but not the right hand) is affected by pictorial illusions that distort the perceived size of the object (Gonzalez et al., 2006). It may also explain why performance in simple visuospatial tasks, such as line bisection, are typically hampered after lesions in the right parietal cortex but not in the left side (Halligan et al., 2003).

\section{Conclusions}

In this study, we demonstrate that external actions are represented in the human anterior intraparietal cortex according to their motor attributes (i.e., the identity of the acting hand). Furthermore, we show that this motor oriented representation is a property of specific visuo-motor areas, which are active during both object-manipulation (without visual feedback) and observation of actions made by others. This unique dual representation, demonstrated on a subject by subject basis, forms a solid support for the mirror system hypothesis. In particular, the specificity of the anterior intraparietal cortex to the identity of the seen hand, as well as to the nature of the viewed grasping, suggests that it is involved in the specific simulation of hand actions.

\section{References}

Aziz-Zadeh L, Koski L, Zaidel E, Mazziotta J, Iacoboni M (2006) Lateralization of the human mirror neuron system. J Neurosci 26:2964-2970.

Binkofski F, Dohle C, Posse S, Stephan KM, Hefter H, Seitz RJ, Freund HJ (1998) Human anterior intraparietal area subserves prehension: a combined lesion and functional MRI activation study. Neurology 50:1253-1259.

Bonda E, Frey S, Petrides M (1996) Evidence for a dorso-medial parietal system involved in mental transformations of the body. J Neurophysiol 76:2042-2048.

Boynton GM, Engel SA, Glover GH, Heeger DJ (1996) Linear systems analysis of functional magnetic resonance imaging in human V1. J Neurosci 16:4207-4221.

Buccino G, Binkofski F, Fink GR, Fadiga L, Fogassi L, Gallese V, Seitz RJ, Zilles K, Rizzolatti G, Freund HJ (2001) Action observation activates premotor and parietal areas in a somatotopic manner: an fMRI study. Eur J Neurosci 13:400-404.

Buccino G, Vogt S, Ritzl A, Fink GR, Zilles K, Freund HJ, Rizzolatti G (2004) Neural circuits underlying imitation learning of hand actions: an eventrelated fMRI study. Neuron 42:323-334.

Cross ES, Hamilton AF, Grafton ST (2006) Building a motor simulation de novo: observation of dance by dancers. Neuroimage 31:1257-1267.
Culham JC, Danckert SL, DeSouza JF, Gati JS, Menon RS, Goodale MA (2003) Visually guided grasping produces fMRI activation in dorsal but not ventral stream brain areas. Exp Brain Res 153:180-189.

Desmurget M, Epstein CM, Turner RS, Prablanc C, Alexander GE, Grafton ST (1999) Role of the posterior parietal cortex in updating reaching movements to a visual target. Nat Neurosci 2:563-567.

Engel SA, Glover GH, Wandell BA (1996) Retinotopic organization in human visual cortex and the spatial precision of functional MRI. Cereb Cortex 7:181-192.

Fogassi L, Ferrari PF, Gesierich B, Rozzi S, Chersi F, Rizzolatti G (2005) Parietal lobe: from action organization to intention understanding. Science 308:662-667.

Forman SD, Cohen JD, Fitzgerald M, Eddy WF, Mintun MA, Noll DC (1995) Improved assessment of significant activation in functional magnetic resonance imaging (fMRI): use of a cluster-size threshold. Magn Reson Med 33:636-647.

Frey SH, Vinton D, Norlund R, Grafton ST (2005) Cortical topography of human anterior intraparietal cortex active during visually guided grasping. Brain Res Cogn Brain Res 23:397-405.

Friston KJ, Holmes AP, Worsley KJ (1999) How many subjects constitute a study? Neuroimage 10:1-5.

Gallese V, Murata A, Kaseda M, Niki N, Sakata H (1994) Deficit of hand preshaping after muscimol injection in monkey parietal cortex. Neuroreport 5:1525-1529.

Gallese V, Fadiga L, Fogassi L, Rizzolatti G (1996) Action recognition in the premotor cortex. Brain 119:593-609.

Gangitano M, Mottaghy FM, Pascual-Leone A (2001) Phase-specific modulation of cortical motor output during movement observation. Neuroreport 12:1489-1492.

Gangitano M, Mottaghy FM, Pascual-Leone A (2004) Modulation of premotor mirror neuron activity during observation of unpredictable grasping movements. Eur J Neurosci 20:2193-2202.

Gonzalez CL, Ganel T, Goodale MA (2006) Hemispheric specialization for the visual control of action is independent of handedness. J Neurophysiol 95:3496-3501.

Halligan PW, Fink GR, Marshall JC, Vallar G (2003) Spatial cognition: evidence from visual neglect. Trends Cogn Sci 7:125-133.

Iacoboni M, Woods RP, Brass M, Bekkering H, Mazziotta JC, Rizzolatti G (1999) Cortical mechanisms of human imitation. Science 286:2526-2528.

Karnath HO, Perenin MT (2005) Cortical control of visually guided reaching: evidence from patients with optic ataxia. Cereb Cortex 15:1561-1569.

Kertzman C, Schwarz U, Zeffiro TA, Hallett M (1997) The role of posterior parietal cortex in visually guided reaching movements in humans. Exp Brain Res 114:170-183.

de Lange FP, Hagoort P, Toni I (2005) Neural topography and content of movement representations. J Cogn Neurosci 17:97-112.

Molnar-Szakacs I, Iacoboni M, Koski L, Mazziotta JC (2005) Functional segregation within pars opercularis of the inferior frontal gyrus: evidence from fMRI studies of imitation and action observation. Cereb Cortex 15:986-994.

Murata A, Gallese V, Luppino G, Kaseda M, Sakata H (2000) Selectivity for the shape, size, and orientation of objects for grasping in neurons of monkey parietal area AIP. J Neurophysiol 83:2580-2601.

Nelissen K, Luppino G, Vanduffel W, Rizzolatti G, Orban GA (2005) Observing others: multiple action representation in the frontal lobe. Science 310:332-336.

Parsons LM, Fox PT, Downs JH, Glass T, Hirsch TB, Martin CC, Jerabek PA, Lancaster JL (1995) Use of implicit motor imagery for visual shape discrimination as revealed by PET. Nature 375:54-58.

Penfield W, Rasmussen T (1952) The cerebral cortex of man. New York: MacMillan.

Rizzolatti G, Fadiga L, Gallese V, Fogassi L (1996) Premotor cortex and the recognition of motor actions. Brain Res Cogn Brain Res 3:131-141.

Rizzolatti G, Fogassi L, Gallese V (2001) Neurophysiological mechanisms underlying the understanding and imitation of action. Nat Rev Neurosci 2:661-670.

Shmuelof L, Zohary E (2005) Dissociation between ventral and dorsal fMRI activation during object and action recognition. Neuron 47:457-470.

Tunik E, Frey SH, Grafton ST (2005) Virtual lesions of the anterior intraparietal area disrupt goal-dependent on-line adjustments of grasp. Nat Neurosci 8:505-511. 